\title{
Primeness in Quantales
}

\author{
$1^{\text {st }}$ Flaulles Boone Bergamaschi \\ Dept. de Ciências Exatas e Tecnológicas \\ Univ. Estadual do Sudoeste da Bahia \\ Vitoria da Conquista, Brasil \\ flaulles@yahoo.com.br
}

\author{
$2^{\text {nd }}$ Regivan H. N. Santiago \\ Dept. de Inf. e Matemática Aplicada \\ Univ. Federal do Rio Grande do Norte \\ Natal, Brasil \\ regivan@dimap.ufrn.br
}

\author{
$3^{\text {rd }}$ Alexsandra Oliveira Andrade \\ Dept. de Ciências Exatas e Tecnologicas \\ Univ. Estadual do Sudoeste da Bahia \\ Vitoria da Conquista, Brasil \\ alexsandra0andrade@gmail.com
}

\begin{abstract}
In this paper we propose a new concept of primeness in quantales. It is proved that this concept coincide with classical definition in commutative quantales, but no longer valid in the noncommutative setting. Also, the notions of strong and uniform strong primeness are investigated.
\end{abstract}

Keywords-Primeness; Prime ideal; Strong; Quantales.

\section{INTRODUCTION}

As it is known, ideals are the main object in the investigation of ring theory and provide important information about the rings because they are structural pieces. The same may occur in quantales. The definition of prime ideals proposed in [1] is based on elements of a quantale and we ponder it is geared to commutative environment. When we move from commutative to the noncommutative setting, elementwise should be replaced by an approach based on ideals. Nevertheless, some authors defined the concept of primeness for commutative and noncommutative cases without realizing that this concept may not be suitable for noncommutative setting as it was well shown by Navarro et. al. in [2]. We state that the concept of prime ideal of general quantales could be defined as it is done in ring theory, i.e. based on ideals.

The main aim of this paper is to study the notion of primeness in the following perspective: we rename prime ideal defined in [1] to completely prime ideal and define a new concept of prime ideal for general quantales. Then we translate an important result in ring theory for quantales environment (theorem 2) to prove that these two concepts coincide in the commutative setting, but are no longer valid in the noncommutative setting (see Proposition 3). Also, based on the studies of Lawrence and Handelman [3], started in 1975 , the notion of strong primeness is investigated for general quantales.

\section{BASIC ON QUANTALES}

This section proposes a new concept of prime ideals suitable for commutative and non-commutative quantales. The definition of prime ideal used in [4] and [1] will be called herein completely prime ideals. We drew attention to the theorem 2 where prime ideals can be characterized in a certain way via elements. The Proposition 4 shows that in the commutative case, prime and completely prime concepts coincide, which are no longer valid in the noncommutative setting according to Proposition 3. Finally, the concept of quantale prime is proposed.
Definition 1: [5] A quantale is a complete lattice $Q$ with an associative binary operation $\circ$ satisfying:

$$
a \circ\left(\bigvee_{k \in K} b_{k}\right)=\bigvee_{k \in K}\left(a \circ b_{k}\right), \quad\left(\bigvee_{k \in K} a_{k}\right) \circ b=\bigvee_{k \in K}\left(a_{k} \circ b\right)
$$

for all $a, b, a_{k}, b_{k} \in Q$ and $k \in K$, where $K$ is a index set.

A quantale $Q$ is called commutative whenever $a \circ b=b \circ a$ for $a, b \in Q$. In this paper we denote the least and greatest elements of a quantale by $\perp$ and $T$ respectively. If there exists an element $e$ in $Q$ such that $x \circ e=e \circ x=x$ for all $x$ in $Q$ the quantale is called a quantale with identity. In this paper we consider quantales with identity.

Definition 2: [4] Let $Q$ be a quantale. A non-empty subset $I \subseteq Q$ is called a right ideal of $Q$ if it satisfies the following conditions:

i) $a, b \in I$ implies $a \vee b \in I$;

ii) for all $a, b \in Q, a \in I$ and $b \leq a$ imply $b \in I$,

iii) for all $x \in Q$ and $a \in I$, we have $a \circ x \in I$.

Similarly we may define left ideal replacing (iii) by: (iii') for all $x \in Q$ and $a \in I$, we have $x \circ a \in I$. If $I$ is both right and left ideal of $Q$, we call $I$ a two-sided ideal or simply an ideal of $Q$.

Clearly by (ii) $\perp \in I$. Also, the set of all ideals of $Q$ is closed under arbitrary intersections.

In $Q$ we denote the subset $I \circ J=\{i \circ j \in Q: i \in$ $I$ and $j \in J\}$ and $A \vee B=\{a \vee b: a \in A$ and $b \in B\}$. Since the operation $\circ$ is associative, we have $(A \circ B) \circ C=A \circ(B \circ C)$. Also, if $A$ is an two-sided ideal, then $A \circ Q, Q \circ A, Q \circ A \circ Q \subseteq$ A.

As usual, $\vee$ induces an order relation $\leq$ on $Q$ by putting $x \leq y \Leftrightarrow x \vee y=y$. Moreover, $\leq$ is a congruence i.e. for every $x, y, u, v \in Q$ if $x \leq y$ and $u \leq v$, then $x \circ u \leq y \circ v$. To prove this, we first observe that if $w \leq z$ then, for any $s \in Q, s \circ w \leq s \circ z$ and $w \circ s \leq z \circ s$ because $z=w \vee z$ implies $s \circ z=s \circ(w \vee z)=(s \circ w) \vee(s \circ z)$ and $z \circ s=$ $(w \vee z) \circ s=(w \circ s) \vee(z \circ s)$; now suppose $x \leq y$ and $u \leq v$, then $x \circ u \leq y \circ u$ and $y \circ u \leq y \circ v$. Hence, $x \circ u \leq y \circ \bar{v}$ by transitivity. 


\section{PRIMENESS}

In what follows we propose a more general definition of prime ideals which encompasses commutative and noncommutative quantales.

Definition 3: A prime ideal in a quantale $Q$ is any proper ideal $P$ such that, whenever $I, J$ are ideals of $Q$ with $I \circ J \subseteq P$, either $I \subseteq P$ or $J \subseteq P$.

Definition 4: A subset $P$ of a quantale $Q$ is called completely prime ideal if $x$ and $y$ are two elements of $Q$ such that their product $x \circ y \in I$, then $x \in I$ or $y \in I$.

As we will see the concept of prime and completely prime ideals are different. But in a commutative quantale this concept is the same.

Proposition 1: If $P$ is completely prime, then $P$ is prime.

Proof: Suppose that $P$ is completely prime and $I \circ J \subseteq P$, but $J \nsubseteq P$, where $I, J$ are ideals of $Q$. Thus, there exists $j \in J$ such that $j \notin P$. For all $i \in I$ we have $i \circ j \in I \circ J \subseteq P$, as $P$ is completely prime and $j \notin P$, then $i \in P$. Therefore $I \subseteq P$.

The Proposition 3 will show that the converse of this Proposition is not true.

Definition 5: [4] Let $Q$ be a quantale and $A \subseteq Q$. The least ideal containing $A$ is called the ideal generated by $A$, and denoted as $\langle A\rangle$.

Clearly, $\langle\emptyset\rangle=\{\perp\}$. If $\emptyset \neq A \subseteq Q$, then we have the following result.

Proposition 2: [4] Let $A$ be a non-empty subset of a quantale $Q$. Then $\langle A\rangle=\{x \in Q: x \leq$ $\bigvee_{i=1}^{n} a_{i}$, for some positive integer $n$ and $a_{1}, \ldots, a_{n} \in A \bar{\cup}$ $(A \circ Q) \cup(Q \circ A) \cup(Q \circ A \circ Q)\}$.

We may denote $\langle a\rangle=\langle\{a\}\rangle$ and $a \circ Q=\{a\} \circ Q$.

Lemma 1: $\langle a\rangle \circ Q \subseteq\langle a\rangle$ for all $a \in Q$. If there exists an unit 1 in $Q$, then $\langle a\rangle \circ Q=\langle a\rangle$.

Proof: Let $x \circ q \in\langle a\rangle \circ Q$, where $x \in\langle a\rangle$ and $q \in Q$. Hence, $x \circ q \leq \bigvee_{i=1}^{n}\left(a_{i} \circ q\right)$, where $a_{i} \circ q \in a \circ Q \cup Q \circ a \cup Q \circ a \circ Q$. Thus, $x \circ q \in\langle a\rangle$. On the other hand if there exists unit 1 in $Q$, we write $z \in\langle a\rangle$ as $z=z \circ 1$. Thus, $z \in\langle a\rangle \circ Q$ and we have $\langle a\rangle \circ Q=\langle a\rangle$.

Theorem 2: For an ideal $P$ in $Q$ the following statements are equivalent:

(1) $P$ is prime ideal;

(2) $\langle a\rangle \circ\langle b\rangle \subseteq P$ implies $a \in P$ or $b \in P$;

(3) $a \circ Q \circ b \subseteq P$ implies $a \in P$ or $b \in P$.

Proof: For $(1) \Rightarrow(2)$ note that $\langle a\rangle$ and $\langle b\rangle$ are ideals of $Q$. As $P$ is prime and $\langle a\rangle \circ\langle b\rangle \subseteq P$, then $\langle a\rangle \subseteq P$ or $\langle b\rangle \subseteq P$. Hence, $a \in P$ or $b \in P$. For $(2) \Rightarrow(1)$, assume that $I \circ \bar{J} \subseteq P$, but $J \nsubseteq P$, where $I, J$ are ideals of $Q$. Thus, there exists $j \in J$ such that $j \notin P$. Given $i \in I$ we have $\langle i\rangle \subseteq I$. Hence, $\langle i\rangle \circ\langle j\rangle \subseteq I \circ J \subseteq P$. By hypothesis $i \in P$ or $j \in P$. As $j \notin P$ then we have $i \in P$. Therefore, $I \subseteq P$.
For (3) $\Rightarrow(1)$, assume that $I \circ J \subseteq P$, but $J \nsubseteq P$, where $I, J$ are ideals of $Q$. Thus, there exists $j \in J$ such that $j \notin P$. Given $i \in I$ we have $i \circ Q \circ j \subseteq I \circ J \subseteq P$. Hence, $i \in P$ or $j \in P$, as $j \notin P$ then we have $i \in P$. Thus, $I \subseteq P$.

For (1) $\Rightarrow(3)$, suppose $a \circ Q \circ b \subseteq P$, we first shall show that $\langle a\rangle \circ Q \circ\langle b\rangle \subseteq P$. For this, let $x \circ q \circ y \in\langle a\rangle \circ Q \circ\langle b\rangle$, where $x \in\langle a\rangle, y \in\langle b\rangle$ and $q \in Q$. Hence, by Proposition 2, $x \leq \vee_{i=1}^{n} a_{i}$ and $y \leq \vee_{j=1}^{m} b_{j}$, where $a_{i} \in(a \circ Q) \cup(Q \circ$ $a) \cup(Q \circ a \circ Q)$ and $b_{i} \in(b \circ Q) \cup(Q \circ b) \cup(Q \circ b \circ Q)$. Hence $x \circ q \circ y \leq\left(\vee_{i=1}^{n} a_{i}\right) \circ q \circ\left(\vee_{j=1}^{m} b_{j}\right)=\left(\vee_{i=1}^{n}\left(a_{i} \circ q\right)\right) \circ$ $\left(\vee_{j=1}^{m} b_{j}\right)=\vee_{i=1}^{n}\left(a_{i} \circ q \circ \vee_{j=1}^{m} b_{j}\right)=\vee_{i=1}^{n}\left(\vee_{j=1}^{m}\left(a_{i} \circ q \circ b_{j}\right)\right)$.

Observe that $a_{i} \in a \circ Q \cup Q \circ a \cup Q \circ a \circ Q$ and $b_{j} \in b \circ Q \cup Q \circ$ $b \cup Q \circ b \circ Q$ it is no hard to see that $a_{i} \circ q \circ b_{j} \in a \circ Q \circ b \subseteq P$ for all $i, j$. As $P$ is an ideal we have $x \circ q \circ y \in P$. Thus, $\langle a\rangle \circ Q \circ\langle b\rangle \subseteq P$. By the Lemma $1\langle a\rangle \circ Q=\langle a\rangle$. Then, $\langle a\rangle \circ Q \circ\langle b\rangle=\langle a\rangle \circ\langle b\rangle \subseteq P$. By the first proof $((1) \Leftrightarrow(2))$ we have $a \in P$ or $b \in P$.

Proposition 3: There exists a noncommutative quantale where a prime ideal is not a completely prime ideal.

\section{Proof:}

Consider $G$ the invertible $2 \times 2$ matrices under multiplication over the real interval $[0,1]$ and the partial or$\operatorname{der} A \leq B \Leftrightarrow a_{i j} \leq b_{i j}$. According to Rosenthal [5], page 19 , example 16 , any complete partially ordered group (written multiplicatively) is a quantale with $a \circ b=a \cdot b$. Thus, $G$ is a noncommutative quantale. Let $\langle 0\rangle$ as an ideal generated by 0 , clearly $\langle 0\rangle=\{0\}$. We will show that the $\langle 0\rangle$ (zero ideal) is prime, but not completely prime by using the Theorem 2 (3). Thus, suppose that $X=\left(\begin{array}{ll}a & b \\ c & d\end{array}\right)$ and $Y=\left(\begin{array}{ll}e & f \\ g & h\end{array}\right)$ are two matrices such that $X \circ G \circ Y \subseteq\langle 0\rangle$. Hence $X \circ T \circ Y=\left(\begin{array}{ll}0 & 0 \\ 0 & 0\end{array}\right)$ for all matrix $T \in G$. Then, in particular,

$$
\begin{aligned}
& X \circ\left(\begin{array}{ll}
1 & 0 \\
0 & 0
\end{array}\right) \circ Y=\left(\begin{array}{ll}
a & b \\
c & d
\end{array}\right)\left(\begin{array}{ll}
1 & 0 \\
0 & 0
\end{array}\right)\left(\begin{array}{ll}
e & f \\
g & h
\end{array}\right)= \\
& \left(\begin{array}{cc}
a e & a f \\
c e & c f
\end{array}\right)=0 \Leftrightarrow a=c=0 \text { or } e=f=0,
\end{aligned}
$$

$X\left(\begin{array}{ll}0 & 1 \\ 0 & 0\end{array}\right) Y=\left(\begin{array}{ll}a & b \\ c & d\end{array}\right)\left(\begin{array}{ll}0 & 1 \\ 0 & 0\end{array}\right)\left(\begin{array}{ll}e & f \\ g & h\end{array}\right)=$ $\left(\begin{array}{cc}a g & a h \\ c g & c h\end{array}\right)=0 \Leftrightarrow a=c=0$ or $g=h=0$,

$$
X \circ\left(\begin{array}{ll}
0 & 0 \\
1 & 0
\end{array}\right) \circ Y=\left(\begin{array}{ll}
a & b \\
c & d
\end{array}\right)\left(\begin{array}{ll}
0 & 0 \\
1 & 0
\end{array}\right)\left(\begin{array}{ll}
e & f \\
g & h
\end{array}\right)=
$$$$
\left(\begin{array}{cc}
b e & b f \\
d e & d f
\end{array}\right)=0 \Leftrightarrow b=d=0 \text { or } e=f=0 \text {, }
$$

$$
\begin{gathered}
X \circ\left(\begin{array}{ll}
0 & 0 \\
0 & 1
\end{array}\right) \circ Y=\left(\begin{array}{ll}
a & b \\
c & d
\end{array}\right)\left(\begin{array}{ll}
0 & 0 \\
0 & 1
\end{array}\right)\left(\begin{array}{ll}
e & f \\
g & h
\end{array}\right)= \\
\left(\begin{array}{cc}
b g & b h \\
d g & d h
\end{array}\right)=0 \Leftrightarrow b=d=0 \text { or } g=h=0,
\end{gathered}
$$


Hence, a solution must verify that $X=\left(\begin{array}{ll}0 & 0 \\ 0 & 0\end{array}\right)$ or $Y=\left(\begin{array}{ll}0 & 0 \\ 0 & 0\end{array}\right)$. Therefore $X \in\langle 0\rangle$ or $Y \in\langle 0\rangle$ and then $\langle 0\rangle$ is prime. Nevertheless, $\langle 0\rangle$ is not completely prime, since

$$
\begin{aligned}
& \left(\begin{array}{ll}
0 & 1 \\
0 & 0
\end{array}\right) \circ\left(\begin{array}{ll}
0 & 1 \\
0 & 0
\end{array}\right)=\left(\begin{array}{ll}
0 & 0 \\
0 & 0
\end{array}\right) \text { although } \\
& \left(\begin{array}{ll}
0 & 1 \\
0 & 0
\end{array}\right) \notin\langle 0\rangle .
\end{aligned}
$$

Proposition 4: In a commutative quantale an ideal is completely prime iff it is prime.

Proof: If $P$ is a completely prime ideal of a quantale $Q$, then by the Proposition $1 P$ is prime. On the other hand, suppose $P$ is a prime ideal and $a \circ b \in P$ for any $a, b \in Q$. Let $x \circ y \in\langle a\rangle \circ\langle b\rangle$, where $x \in\langle a\rangle$ and $y \in\langle b\rangle$. Thus, $x \circ y \leq \bigvee_{i=1}^{n} a_{i} \circ \bigvee_{j=1}^{m} b_{j}=\bigvee_{i=1}^{n}\left(\bigvee_{j=1}^{m}\left(a_{i} \circ b_{j}\right)\right)$, where $a_{i} \in a \circ Q \cup Q \circ a \cup Q \circ a \circ Q$ and $b_{j} \in b \circ Q \cup Q \circ b \cup Q \circ b \circ Q$. As $Q$ is commutative $a \circ Q=Q \circ a=Q \circ a \circ Q$ and $b \circ Q=Q \circ b=Q \circ$ $b \circ Q$. Thus, $a_{i} \circ b_{j} \in a \circ Q \circ b \circ Q=a \circ b \circ Q$ for all $i=1, \ldots, n$ and $j=1, \ldots, m$. Hence, $a_{i} \circ b_{j}=a \circ b \circ q \in P$ and then $x \circ y \leq \bigvee_{i=1}^{n}\left(\bigvee_{j=1}^{m}\left(a_{i} \circ b_{j}\right)\right) \in P$. Therefore, $\langle a\rangle \circ\langle b\rangle \subseteq P$ and by the Theorem 2 we have $a \in P$ or $b \in P$.

In what follows, we will introduce the notion of quantale prime. As we know, in ring theory, a quantale is prime iff the ideal generated by 0 is a prime ideal. Then, the Proposition 5 translates this result into quantale environment.

Definition 6: A quantale $Q$ is called prime if given $a, b \in$ $Q$ with $a \neq \perp$ and $b \neq \perp$, there exists $f \in Q$ such that $a \circ f \circ b \neq \perp$. ideal.

Proposition 5: A quantale $Q$ is prime iff $\langle\perp\rangle$ is a prime

Proof: Suppose $Q$ prime and assume that $I \circ J \subseteq\langle\perp\rangle$, but $I, J \nsubseteq\langle\perp\rangle$, where $I, J$ are ideals of $Q$. Thus, there exists $i \in I, j \in J$ such that $i, j \neq \perp$. As $Q$ is prime, there exists $q \in Q$ such that $i \circ q \circ j \neq \perp$, then we have a contradiction because $i \circ q \circ j \in I \circ J \subseteq\langle\perp\rangle$. Hence, $I \subseteq\langle\perp\rangle$ or $J \subseteq\langle\perp\rangle$. On the other hand, suppose $\langle\perp\rangle$ is a prime ideal of $Q$. Given $a, b \neq \perp$ in $Q$, suppose $a \circ q \circ b=\perp$ for all $q \in Q$. Hence, $a \circ Q \circ b \subseteq\langle\perp\rangle$. As $\langle\perp\rangle$ is a prime ideal, then $a \in\langle\perp\rangle$ or $b \in\langle\perp\rangle$, but $a, b \neq \perp$.

\section{STRONG PRIMENESS IN QUANTALES}

Strongly prime rings were introduced in 1973, as a prime ring with finite condition in the generalization of results on group rings proved by Lawrence in [3]. In this section we bring this concept to quantales making specific adaptations for this environment.

Definition 7: Let $A$ be a subset of a quantale $Q$. The right annihilator of $A$ is defined as $A n_{r}(A)=\{x \in Q: A x=$ $\langle\perp\rangle\}$. Similarly, we can define the left annihilator $A n_{l}$.

Definition 8: [3] A quantale $Q$ is called right strongly prime if for each $x \in Q-\{\perp\}$ there exists a finite nonempty subset $F_{x}$ of $Q$ such that $A n_{r}\left(x \circ F_{x}\right)=\langle\perp\rangle$. Clearly if $Q$ is right strongly prime, then $Q$ is prime. The set $F_{x}$ is called an insulator of $x$ in $Q$.

Proposition 6: If $Q$ is right strongly prime, then every nonzero ideal $I$ of $Q$ contains a finite subset $F$ which has right annihilator zero.

Proof: Suppose $Q$ right strongly prime. Let $x \in I$ and $x \neq \perp$ and $F=x \circ F_{x} \subseteq I$. Thus, $A n_{r}(F)=\langle\perp\rangle$.

It is clear that every right strongly prime quantale is a prime quantale. It is also possible to define left strongly prime in a similar manner for right strong primeness.

Definition 9: A quantale $Q$ is called uniformly strongly prime (usp) if the same right insulator may be chosen for each nonbottom element.

Proposition 7: A quantale $Q$ is a right uniformly strongly prime iff there exists a finite subset $F \subseteq Q$ such that for any two nonbottom elements $x$ and $y$ of $Q$, there exists $f \in F$ such that $x \circ f \circ y \neq \perp$.

Proof: Let $Q$ be uniformly right strongly prime quantale. Hence $Q$ has a uniform right insulator $F$ which is a finite set such that for any element $x \in Q, x \circ F$ has no nonbottom right annihilators. Thus, if $x$ and $y$ are any two nonbottom elements in $Q, y$ cannot be in the annihilator of $x \circ F$. Hence there is an $f \in F$ such that $x \circ f \circ y \neq \perp$. For the reverse implication it is easy to see that if the condition is satisfied then for any $x \neq \perp$ in $Q$, no nonbottom element annihilates $x \circ F$ on the right. Hence $Q$ is uniformly right strongly prime

It is clear that the condition in Proposition 7 is not one-sided; consequently, this condition is also equivalent to uniformly left strongly prime, and we have:

Corollary 3: $Q$ is uniformly right strongly prime iff $Q$ is uniformly left strongly prime.

Corollary 4: A quantale $Q$ is uniformly strongly prime iff there exists a finite subset $F \subseteq Q$ such that $a \circ F \circ b=\perp$ implies $a=\perp$ or $b=\perp$ for all $a, b \in Q$.

Proof: Straightforward.

Definition 10: An ideal $P \neq\langle\perp\rangle$ of a quantale $Q$ is called uniformly strongly prime (usp) ideal if there exists a finite subset $F \subseteq Q$ such that $a \circ F \circ b \subseteq P$ implies $a \in P$ or $b \in P$.

Proposition 8: An ideal $I$ of a quantale $Q$ is a usp ideal iff there exists a finite subset $F \subseteq Q$ such that for any two elements $a, b \in Q \backslash I$ (complement of $I$ in $Q$ ), there exists $f \in F$ such that $x \circ f \circ y \notin I$.

Proof: Suppose $I$ a usp ideal of $Q$. If $a \notin I$ and $b \notin I$ by Definition $10 a \circ F \circ b$ is not a subset of $I$. Hence, there exists $f \in F$ such that $a \circ f \circ b \notin I$. For the converse, note that by hypothesis it is impossible to have $a \circ F \circ b \subseteq I$ and $a \notin I$ and $b \notin I$. 


\section{Final REMARKS}

Prime ideals have developed an important role in ring theory and have attracted the attention of some researchers in the investigation of quantales. As prime ideals are structural pieces of a ring it is relevant to study its concept in order to establish a well-founded quantale theory. With this in mind, it is necessary to investigate primeness over arbitrary quantales, i.e. commutative and noncommutative setting. Therefore, this paper invites the reader to think about primeness in quantales bringing a new perspective for prime ideals.

\section{REFERENCES}

[1] Lingyun Yang and Luoshan Xu. Roughness in quantales. Information Sciences, 220:568-579, 2013.

[2] Gabriel Navarro, Oscar Cortadellas, and FJ. Lobillo. Prime fuzzy ideals over noncommutative rings. Fuzzy Sets and Systems, 199(0):108 - 120, 2012.

[3] David Handelman and John Lawrence. Strongly prime rings. Transactions of the American Mathematical Society, 211:209-223, 1975.

[4] Qingjun Luo and Guojun Wang. Roughness and fuzziness in quantales. Information Sciences, 271:14-30, 2014.

[5] Kimmo I Rosenthal. Quantales and their applications, volume 234. Longman Scientific \& Technical Harlow, 1990. 